\title{
Observation of chaotic polarization attractors from a graphene mode locked soliton fiber laser
}

\author{
Chang Zhao (赵畅) ${ }^{1}$, Qianqian Huang(黄千千) ${ }^{1}$, Mohammed Al Araimi², Aleksey Rozhin ${ }^{3}$, Sergey \\ Sergeyev $^{3}$ and Chengbo Mou（牟成博）*1 \\ ${ }^{1}$ Key Laboratory of Specialty Fiber Optics and Optical Access Networks, Shanghai Institute for Advanced Communication and Data Science, \\ Joint International Research Laboratory of Specialty Fiber Optics and Advanced Communication, Shanghai University, Shanghai 200444, \\ China \\ ${ }^{2}$ Higher College of Technology, Al-Khuwair, PO Box 74, Postal Code 133, Sultanate of Oman \\ ${ }^{3}$ Aston Institute of Photonic Technologies (AIPT), Aston University, Birmingham, B4 7ET, United Kingdom \\ *Corresponding author: mouc1@shu.edu.cn \\ Received Month X, XXXX; accepted Month X, XXXX; posted online Month X, XXXX
}

\begin{abstract}
We have demonstrated an all-fiber passively mode locked erbium-doped fiber laser (EDFL) based on graphene polyvinyl alcohol (PVA) film. By watchfully adjusting polarization controller (PC), two different polarization attractors including polarization locked vector solitons (PLVSs) and circular attractor can be observed. This is first time to explore the dynamics polarization attractors exhibited by vector soliton generated from an EDFL based on graphene.

OCIS Codes: 260.5430, 140.0140, 160.0160, 320.0320.

doi:10.3788/COLXXXXXX.XXXXXX.
\end{abstract}

Vector solitons(VSs) have been extensively studied since the propagation of optical pulses in birefringent fibers were theoretically analyzed in 1987[1]. Soon afterwards, VSs were investigated and defined by Christodoulides et al in birefringent nonlinear dispersive media for the first time ${ }^{[2]}$. VS is a type of stable-state wave containing two orthogonal polarization components which keep polarization fixed or periodic evolution when propagating in a single mode fiber (SMF) that holds birefringence. Consequently, SMF can be expected as an ideal vehicle to propagate VSs as a result of the weak birefringence. It is well known that fiber lasers have the ability to generate VSs, utilizing physical saturable absorbers (SAs) such as semiconductor saturable absorption mirrors (SESAMs), carbon nanotube, graphene etc. Besides, recently years some new 2 Dmaterials such as black phosphorus ${ }^{[3,4]}$, MXene ${ }^{[5]}$, Bismuthene ${ }^{[6]}$ have been reported to serve as excellent SAs.

Due to the nonlinear coupling between the two orthogonal polarization components, various types of VSs can be formed in the laser cavity. In PLVSs, the state of polarization $(\mathrm{SOP})$ and phase velocity are fixed ${ }^{[7,8]}$, while group velocity locked vector solitons (GVLVSs) require the group velocity locking and no demand for phase velocity locking ${ }^{[9]}$. Besides, some periodical propagating VSs can be locked to the cavity roundtrip time or multiplies, which are commonly referred as polarization rotation vector solitons $(\mathrm{PRVSS})[10,11]$. Some researchers have reported the observation of vector nature of various dynamic patterns based on figure-eight fiber lasers ${ }^{[12,13]}$. In the past decade, VSs in passively mode locked fiber lasers using nanomaterials based SAs have been extensively studied. For instance, polarization dynamics of VSs in the cavity utilizing carbon nanotubes (CNTs) have been systematically explored ${ }^{[14-18]}$. Besides, the iconic 2D material such as graphene possesses not only amazing electronic properties but also fantastic optical properties with the potential of operating in a wideband range as a novel SA $[19,20]$. SAs based on graphene also features polarization independent saturable absorption ${ }^{[21]}$. Therefore, for fiber lasers using graphene, intensive investigations have been carried out to study various polarization dynamic patterns and the interaction between two orthogonal polarization components of VSs using polarization beam splitter (PBS) $119,21-24]$ Nevertheless, among all the studies, the phase difference between two orthogonal polarization components and degree of polarization (DOP) are not identified, which can actually be measured using a commercial polarimeter. The missing phase information resulting from the polarization resolved spectral analysis using PBS may lead to ambiguous explanation of VSs. Moreover, the tendency of evolution of VSs at a slow time scale from a small number to thousands of roundtrips is defined as asymptotic states (attractors)

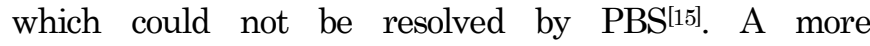
comprehensive interpretation of the dynamic evolution of VSs is therefore necessary.

In this work, as far as we know, we report the polarization attractors for the first time exhibited by VSs operation of EDFL based on graphene-PVA SA. We have experimentally shown two types of polarization attractors with the single pulse regime. The radio frequency (RF) spectrum indicates $\sim 40 \mathrm{~dB}$ signal noise ratio (SNR) at the fundamental frequency. The demonstrated laser may find applications in secure communication ${ }^{[25]}$, manipulation of atoms and nanoparticles ${ }^{[26-28]}$, control of magnetization ${ }^{[29]}$.

Fig.1 shows the typical setup of graphene-based passively mode locked EDFL. The total cavity length is $35.7 \mathrm{~m}$, corresponding to the fundamental frequency of 5.84 
MHz. An $80 \mathrm{~cm}$ highly doped erbium-doped fiber (EDF, OFS EDF80) with a group velocity dispersion of +66.1 $\mathrm{ps}^{2} / \mathrm{km}$ is made use of gain medium in the cavity. In addition, the rest of fiber is SMF with an anomalous dispersion of $-22 \mathrm{ps}^{2} / \mathrm{km}$. The total cavity dispersion of the laser is $-0.7 \mathrm{ps}^{2}$ contributing the formation of conventional soliton. A $980 \mathrm{~nm}$ laser module (OV LINK) used to produce pump light is injected into laser cavity through a 980/1550 $\mathrm{nm}$ wavelength division multiplexer (WDM). The polarization independent isolator (ISO) in the cavity possesses the ability to guarantee the pulse transmit in a unidirectional manner. In addition, reflection from the light existing out the cavity needs to be eliminated by laying an extra ISO outside the cavity. The graphene-PVA film is sandwiched between two fiber connectors to act as a mode locker with the damage threshold of $\sim 380 \mathrm{~mW}$. In-line PC2 is used to control the polarization of the cavity or obtain various polarization attractors, and PC1 connected with pump is involved to optimize cavity anisotropy. Half of the pulse energy is used for intracavity circulation by applying an optical coupler (OC) while the other is implemented for output. Pulse characteristics are detected by an optical spectrum analyzer (OSA, Yokogawa AQ6370C) with a maximum resolution of $0.02 \mathrm{~nm}, 8 \mathrm{GHz}$ oscilloscope (KEYSIGHT DSO90804A) and $3.2 \mathrm{GHz}$ signal analyzer (SSA, 3032X) connected with $12.5 \mathrm{GHz}$ photodetector (Newport 818-BB-51F). Pulse duration of the pulses can be identified by a commercial autocorrelator (FEMTOCHROME, FR-103WS). Furthermore, a commercial polarimeter (THORLABS, IPM5300) is used with $1 \mu \mathrm{s}$ resolution and the distinct attractor can be achieved through 1024 sample points which are characterized by normalized Stokes parameters $S_{1}, S_{2}, S_{3}$ and DOP. Output powers of two polarization component $|u|^{2}$ and $|v|^{2}$, and phase difference $\Delta \varphi$ are related as follows:

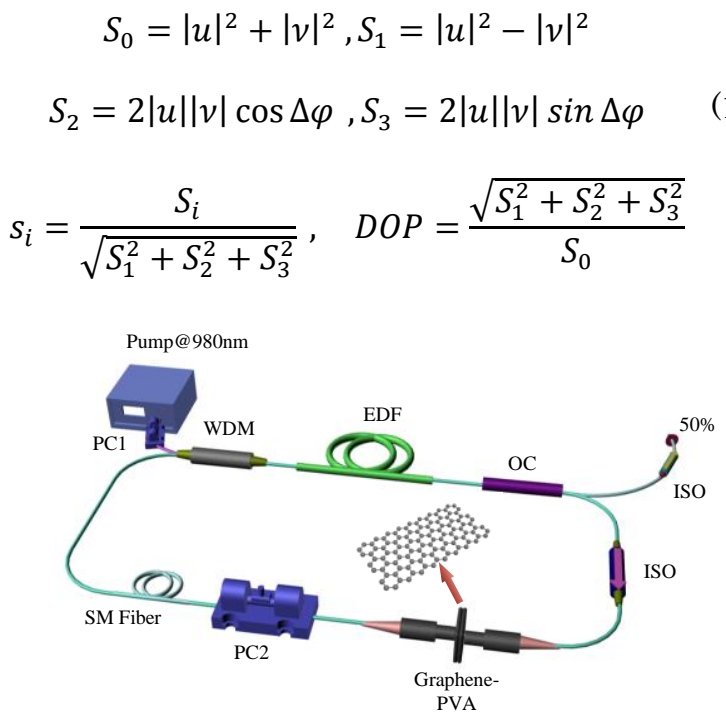

Fig. 1. Schematic setup of the proposed graphene-PVA film mode-locked EDFL.
For preparation of the graphene SA, ultrasonication of graphite is implemented to get dispersions in deionized (DI) water using NanoRuptor processor with sodium deoxycholate (SDC). Then the dispersion is centrifuged (Beckman Coulter Optima Max-XP) followed by mixing with the PVA aqueous solution. The mixing dispersion is then vacuum evaporated at $20^{\circ} \mathrm{C}$ for a few days, leading to the formation of the composite with $50 \mu \mathrm{m}$ thickness.
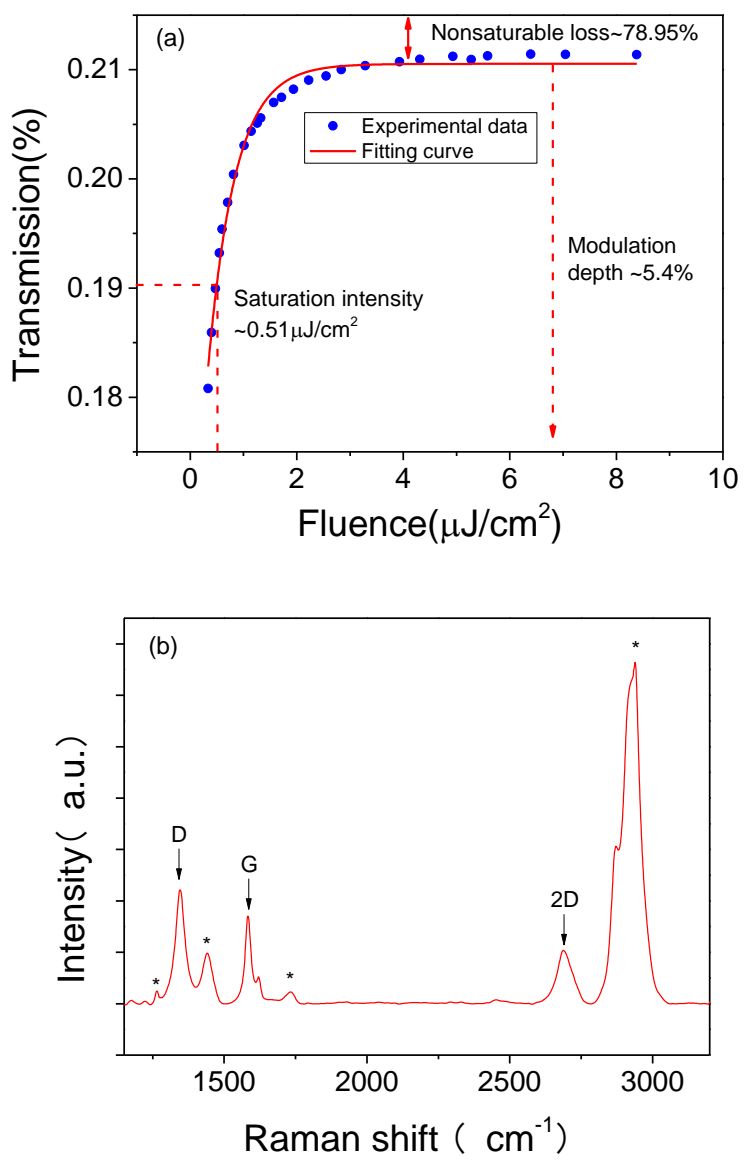

Fig. 2. (a) Nonlinear transmission of graphene SA, (b) Raman spectrum of the graphene SA at $532 \mathrm{~nm}$ excitation.

Fig.2. (a) shows the nonlinear transmission of graphenefilm. The modulation depth, saturable intensity and nonsaturable loss are $5.4 \%, 0.51 \mu \mathrm{J} / \mathrm{cm}^{2}$ and $78.95 \%$ respectively. Fig.2. (b) indicates that the Raman spectroscopic measurement of the produced film which is obtained under the excitation of $532 \mathrm{~nm}$. Ubiquitous G peak of graphene in the Raman spectrum caused by the inplane vibration of $\mathrm{sp}^{2}$-hybrization carbon with the symmetry of $\mathrm{E}_{2 \mathrm{~g}}$ is shown in the picture. The shift of prominent $\mathrm{G}$ and $2 \mathrm{D}$ peaks are 1583 and $2688 \mathrm{~cm}^{-1}$ respectively with the intensity ratio $\left(\mathrm{I}_{2 \mathrm{D}} / \mathrm{I}_{\mathrm{G}}\right)$ of 1.3 corresponding to the formation of few-layer graphene ${ }^{[30]}$. The $\mathrm{D}$ peak generated owing to double-resonance process indicates structural disorder, defects as a fingerprint ${ }^{[31]}$. The stars observed from Fig.2. (b) could be assigned to diffraction peaks associated with the PVA films ${ }^{[32]}$. 

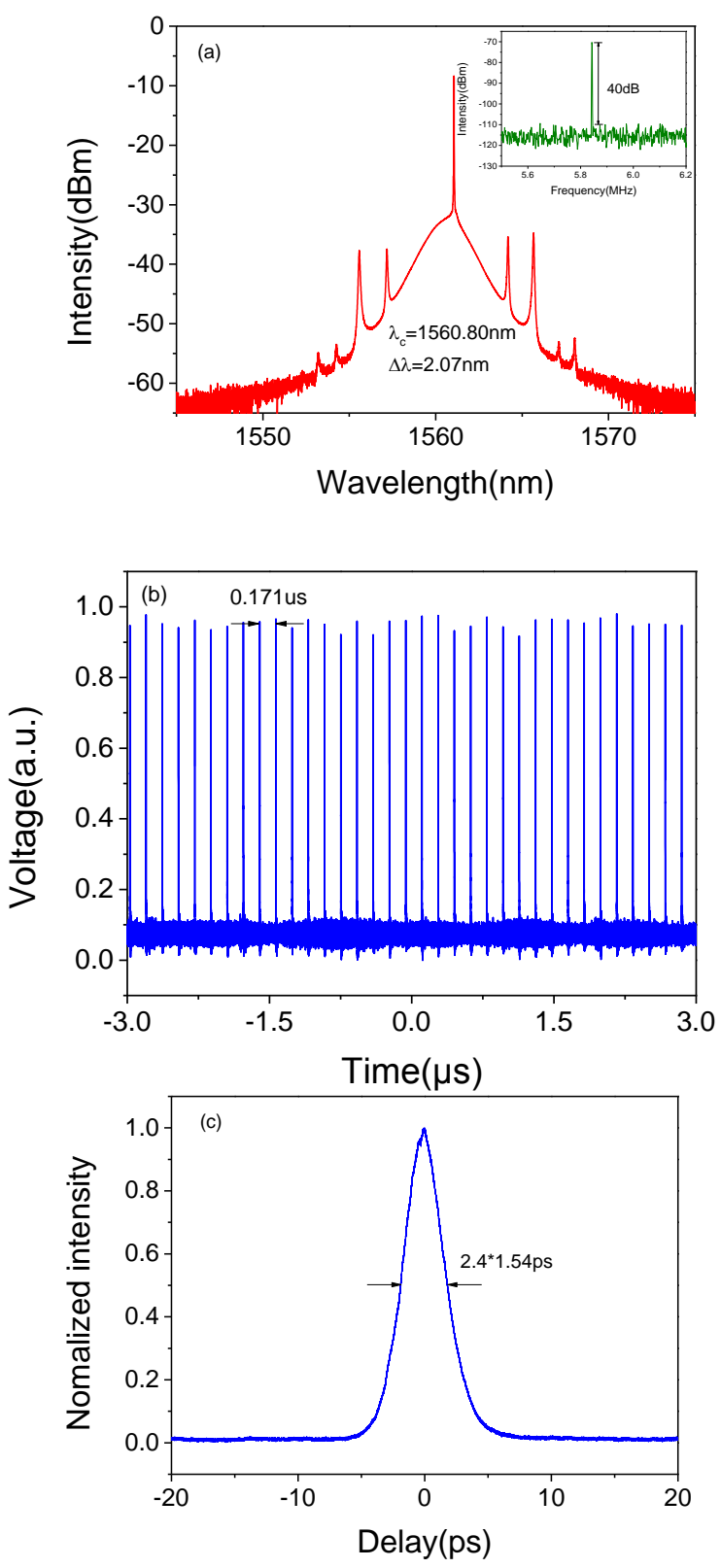

Fig. 3. (a) Optical spectrum, the inset shows the Signal-to-noise ratio (SNR) at fundamental frequency, (b) oscilloscope trace, (c) autocorrelation trace

The ability of the laser has been measured by invariance of pulse and optical spectrum for more than 20 hours. We can obtain mode locking under the pump power of $9.7 \mathrm{~mW}$. While by increasing pump power, we find that single pulse can be still maintained under the power of $11.6 \mathrm{~mW}$ with a strong CW component. PLVSs can be found at the same time. What is more, the maximum pulse energy we can obtain is $\sim 0.1 \mathrm{~nJ}$. Optical spectrum of PLVS is shown in Fig. 3. (a). The pulses center at $1560.6 \mathrm{~nm}$ with the $3 \mathrm{~dB}$ bandwidth of $1.63 \mathrm{~nm}$. Obviously, appearance of Kelly sidebands affirms that the laser operates in the conventional soliton regime. The inset in Fig. 3. (a) suggests a SNR value of $40 \mathrm{~dB}$. Fig. 3. (b) is the oscilloscope trace, where the interval between two pulses is $0.171 \mu \mathrm{s}$ corresponding to the fundamental frequency of $5.84 \mathrm{MHz}$. However, the sampling rate of polarimeter is $1 \mathrm{MHz}$ that is less than 5.84MHz leading to obtained results are averaged conclusion. The autocorrelation measurement illustrated in Fig. 3. (c) shows that the pulse duration is $\sim 2.4 \mathrm{ps}$. The output light is divided into two beams through a 50:50 coupler for simultaneous observation of optical spectrum and state of polarization. The measured total output power and powers of orthogonal components are shown in Fig. 4. (a). Fig. 4. (b) shows that more than $90 \%$ DOP indicates stable output SOP and a fixed phase difference of $\sim 1.2 \mathrm{rad}$, implying the formation of PLVS. Such attractor on the Poincare sphere in the shape of a focused point is depicted in Fig. 4. (c). The complete information of a PLVS is then presented.
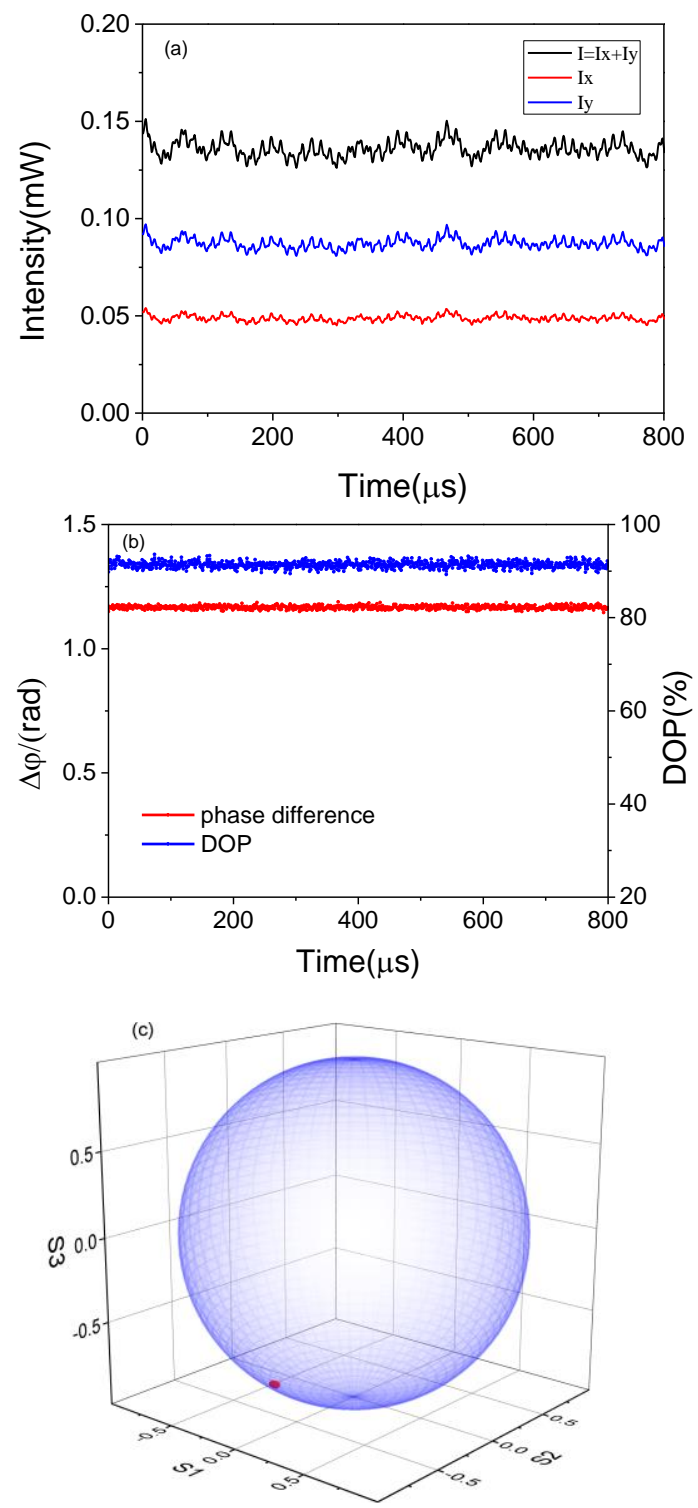

Fig. 4. (a) Optical power of orthogonal component, (b) phase difference and DOP, (c) normalized Stokes parameters shown by Poincare sphere. 

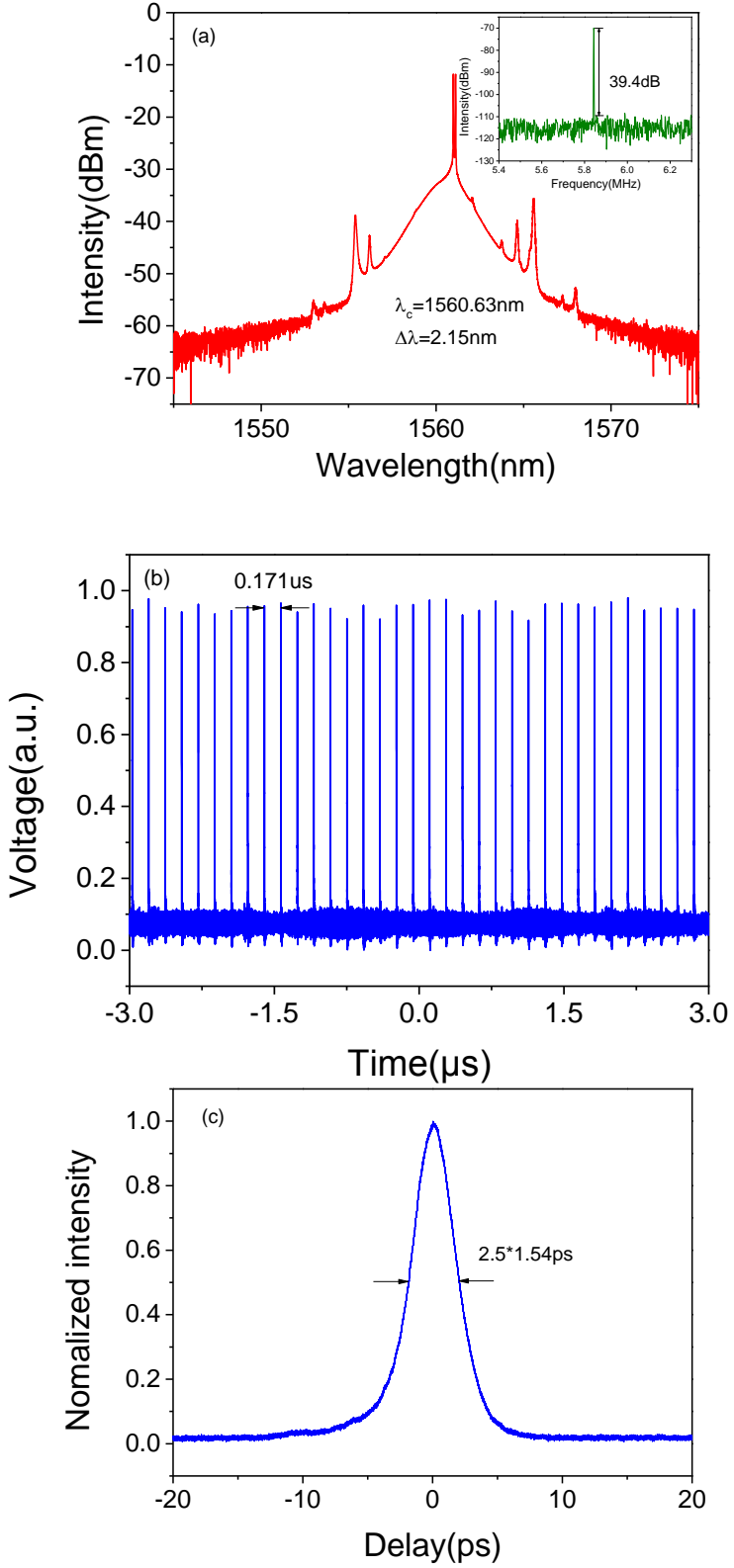

Fig. 5. (a) Optical spectrum, the insert map shows the SNR at fundamental frequency, (b) oscilloscope trace, (c) autocorrelation trace.

We then observe a different attractor during the process of adjusting PC, the optical spectrum is almost identical. Under the condition, $3 \mathrm{~dB}$ bandwidth of optical spectrum is $1.98 \mathrm{~nm}$, period is $0.171 \mu \mathrm{s}$, pulse duration is $2.5 \mathrm{ps}$ which is slightly larger than that shown in Fig. 3. (c). The inset in Fig. 5. (a) demonstrates that SNR is $39.4 \mathrm{~dB}$, which indicates stability of the attractor (Fig. 5. (a-c)). Optical powers of two orthogonal polarized states and total power are indicated in Fig. 6. (a). Obviously, power of polarization along $\mathrm{y}$-axis is similar to total power while that of polarization along $\mathrm{x}$-axis has a very low power. It is found that DOP of the pulse oscillated around $30 \%$ with fast phase difference oscillation as shown in Fig. 6. (b). The resulting trajectory that is winding around a circle on the
Poincare sphere is depicted in Fig. 6. (c). We speculate that the low DOP is a result of the fast changing of SOP. This claims the chaotic polarization attractor on the polarization domain represented by Poincare sphere.

The formation of different attractors can be attributed to the existence of coherent coupling between two orthogonal polarization components. Pump power, amplitude and anisotropy of cavity determine the degree of coupling that controls the formation of polarization attractors. Hence, the polarization attractors shown in Fig. 4 and 6 are caused by increased amplitude and phase anisotropy in the cavity. However, other complex behavior that is not observed in our present work can be the consequence of weaker coupling caused by isotropic cavity ${ }^{[14]}$. It is worthy mention that, during our experiment, there is no Q-switching observed, indicating a high quality of graphene SA.
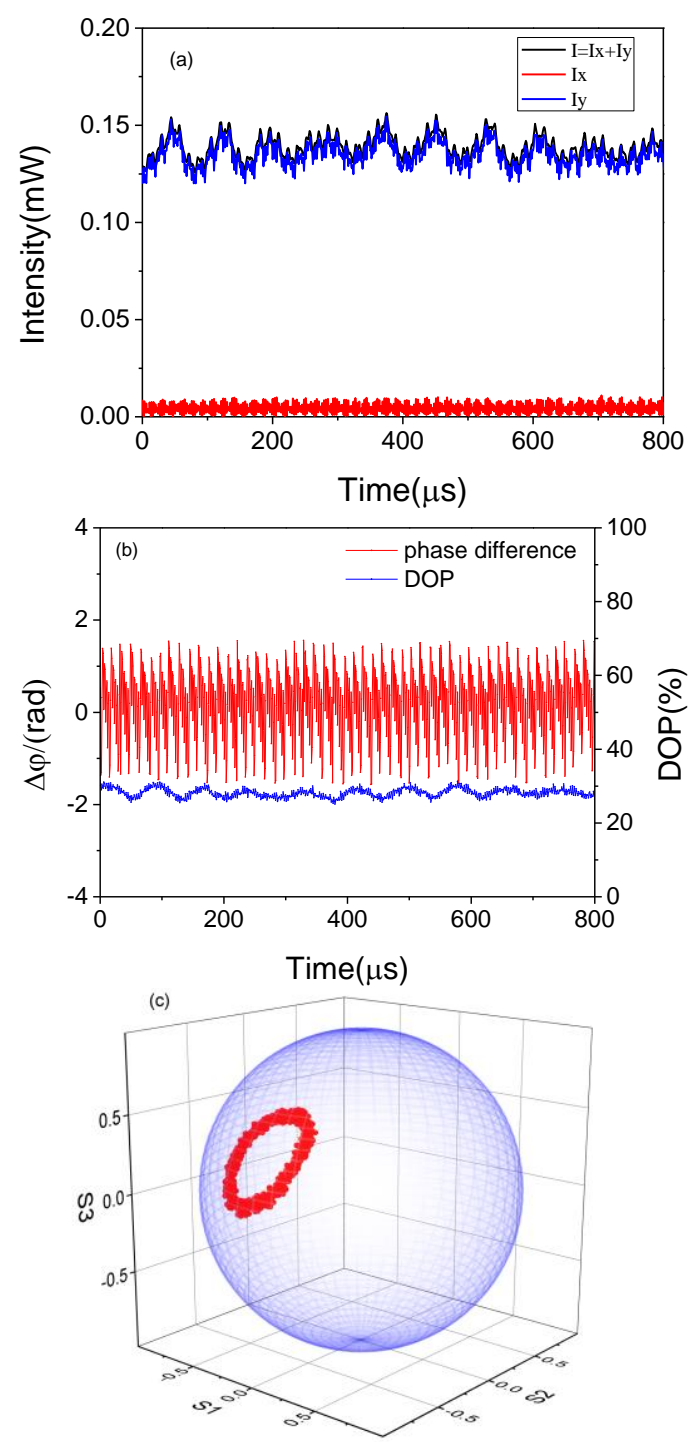

Fig. 6. (a) Optical power of orthogonal component, (b) phase difference and DOP, (c) normalized Stokes parameters shown by Poincare sphere. 
In conclusion, we firstly studied polarization attractor formed by VSs operation of EDFL based on graphene PVAfilm. Under the power of $11.6 \mathrm{~mW}$, two types of polarization attractors can be obtained by carefully adjusting PCs. One attractor is PLVS corresponding to fixed point on the Poincare sphere with a high DOP of $>90 \%$, the other attractor is an elliptical trajectory on the Poincare sphere exhibiting the jump of phase difference between two orthogonal polarization states. The demonstrated work may help in understanding nonlinear optical dynamics in nanomaterial based ultrafast lasers. It may also provide a neat platform for studying nonlinear optical properties of $2 \mathrm{D}$ materials.

The authors would like to acknowledge the National Natural Science Foundation of China (NSFC) (61605107); Young Eastern Scholar Program at Shanghai Institutions of Higher Learning (QD2015027) and support of Young 1000 Talent Plan Program of China. the Open Program (Grant No. 2017GZKF17) of the State Key Laboratory of Advanced Optical Communication Systems and Networks at Shanghai Jiaotong University, China. The Open Fund of IPOC2017B010 (BUPT) and RAEng/The Leverhulme Trust Senior Research Fellowships (LTSRF1617/13/57).

\section{References}

1. C. R. Menyuk, "Stability of solitons in birefringent optical fibers. I: equal propagation amplitudes," Optics Letters 12, 614 (1987).

2. D. N. Christodoulides and R. I. Joseph, "Vector solitons in birefringent nonlinear dispersive media," Optics Letters 13, 53 (1988).

3. L. Zhi-Chao, L. Meng, G. Zhi-Nan, J. Xiao-Fang, L. Ai-Ping, Z. Chu-Jun, Y. Xue-Feng, X. Wen-Cheng, and Z. Han, "Microfiberbased few-layer black phosphorus saturable absorber for ultra-fast fiber laser," Optics Express 23, 20030 (2015).

4. G. Hu, T. Albrow-Owen, X. Jin, A. Ali, Y. Hu, R. C. T. Howe, K. Shehzad, Z. Yang, X. Zhu, R. I. Woodward, T. C. Wu, H. Jussila, J. B. Wu, P. Peng, P. H. Tan, Z. Sun, E. J. R. Kelleher, M. Zhang, Y. $\mathrm{Xu}$, and $\mathrm{T}$. Hasan, "Black phosphorus ink formulation for inkjet printing of optoelectronics and photonics," Nat Commun 8, 278 (2017).

5. X. Jiang, S. Liu, W. Liang, S. Luo, Z. He, Y. Ge, H. Wang, R. Cao, F. Zhang, and Q. Wen, "Broadband Nonlinear Photonics in FewLayer MXene Ti 3 C 2 T x (T = F, O, or OH)," Laser \& Photonics Reviews 12, 1700229 (2017).

6. L. Lu, Z. Liang, L. Wu, Y. X. Chen, Y. Song, S. C. Dhanabalan, J. S. Ponraj, B. Dong, Y. Xiang, and F. Xing, "Few-layer Bismuthene: Sonochemical Exfoliation, Nonlinear Optics and Applications for Ultrafast Photonics with Enhanced Stability," Laser \& Photonics Reviews 12, 1700221 (2018).

7. S. T. Cundiff, B. C. Collings, N. N. Akhmediev, J. M. SotoCrespo, K. Bergman, and W. H. Knox, "Observation of PolarizationLocked Vector Solitons in an Optical Fiber," Physical Review Letters 82, 3988 (1999).

8. B. C. Collings, S. T. Cundiff, N. N. Akhmediev, J. M. Sotocrespo, K. Bergman, and W. H. Knox, "Polarization-locked temporal vector solitons in a fiber laser: experiment," Journal of the Optical Society of America B 17, 354 (2000).

9. D. Y. Tang, H. Zhang, L. M. Zhao, N. Xiang, and X. Wu, "Soliton trapping in fiber lasers," Optics Express 16, 9528 (2008).
10. L. M. Zhao, D. Y. Tang, H. Zhang, and X. Wu, "Polarization rotation locking of vector solitons in a fiber ring laser," Optics Express 16, 10053 (2008).

11. J. H. Wong, K. Wu, H. H. Liu, C. Ouyang, H. Wang, S. Aditya, P. Shum, S. Fu, E. J. R. Kelleher, A. Chernov, and E. D. Obraztsova, "Vector solitons in a laser passively mode-locked by single-wall carbon nanotubes," Optics Communications 284, 2007 (2011).

12. Y. Ling, L. Xueming, and M. Dong, "Observation of dualwavelength dissipative solitons in a figure-eight erbium-doped fiber laser," Optics Express 20, 20992 (2012).

13. Q. Y. Ning, H. Liu, X. W. Zheng, W. Yu, A. P. Luo, X. G. Huang, Z. C. Luo, W. C. Xu, S. H. Xu, and Z. M. Yang, "Vector nature of multi-soliton patterns in a passively mode-locked figure-eight fiber laser," Opt Express 22, 11900 (2014).

14. S. V. Sergeyev, C. Mou, A. Rozhin, and S. K. Turitsyn, "Vector solitons with locked and precessing states of polarization," Optics Express 20, 27434 (2012).

15. C. Mou, S. V. Sergeyev, A. G. Rozhin, and S. K. Turitsyn, "Bound state vector solitons with locked and precessing states of polarization," Opt Express 21, 26868 (2013).

16. V. Tsatourian, S. V. Sergeyev, C. Mou, A. Rozhin, V. Mikhailov, B. Rabin, P. S. Westbrook, and S. K. Turitsyn, "Polarisation dynamics of vector soliton molecules in mode locked fibre laser," Sci Rep 3, 3154 (2013).

17. T. Habruseva, S. Sergeyev, and S. Turitsyn, "Polarization attractors in harmonic mode-locked fiber laser with carbon nanotubes," in Lasers and Electro-Optics, (2014), 1.

18. S. V. Sergeyev, C. Mou, E. G. Turitsyna, A. Rozhin, S. K. Turitsyn, and K. Blow, "Spiral attractor created by vector solitons," Light Science \& Applications 3(2014).

19. H. Zhang, D. Tang, L. Zhao, Q. Bao, and K. P. Loh, "Vector dissipative solitons in graphene mode locked fiber lasers," Optics Communications 283, 3334 (2010).

20. D. Y. Tang, G. Q. Xie, H. H. Yu, H. J. Zhang, J. Ma, J. Y. Wang, L. J. Qian, P. Lv, P. Yuan, and W. L. Gao, "Graphene mode-locked femtosecond laser at $2 \mu \mathrm{m}$ wavelength," Optics Letters 37, 2085 (2012).

21. Y. F. Song, L. Li, H. Zhang, D. Y. Shen, D. Y. Tang, and K. P. Loh, "Vector multi-soliton operation and interaction in a graphene mode-locked fiber laser," Opt Express 21, 10010 (2013).

22. Y. F. Song, H. Zhang, D. Y. Tang, and D. Y. Shen, "Polarization rotation vector solitons in a graphene mode-locked fiber laser," Optics Express 20, 27283 (2012).

23. Y. F. Song, L. Li, D. Y. Tang, and D. Y. Shen, "Quasi-periodicity of vector solitons in a graphene mode-locked fiber laser," Laser Physics Letters 10, 125103 (2013).

24. M. Han, S. Zhang, X. Li, H. Zhang, H. Yang, and T. Yuan, "Polarization dynamic patterns of vector solitons in a graphene modelocked fiber laser," Opt Express 23, 2424 (2015).

25. G. D. VanWiggeren and R. Roy, "Communication with dynamically fluctuating states of light polarization," Phys Rev Lett 88, 097903 (2002).

26. M. Spanner, K. M. Davitt, and M. Y. Ivanov, "Stability of angular confinement and rotational acceleration of a diatomic molecule in an optical centrifuge," Journal of Chemical Physics 115, 8403 (2001).

27. Y. Jiang, T. Narushima, and H. Okamoto, "Nonlinear optical effects in trapping nanoparticles with femtosecond pulses," Nature Physics 6, 1005 (2010).

28. L. Tong, V. D. Miljkovic, and M. Kall, "Alignment, rotation, and spinning of single plasmonic nanoparticles and nanowires using polarization dependent optical forces," Nano Lett 10, 268 (2010).

29. N. Kanda, T. Higuchi, H. Shimizu, K. Konishi, K. Yoshioka, and M. Kuwata-Gonokami, "The vectorial control of magnetization by light," Nat Commun 2, 362 (2011). 
30. H. Zhou, F. Yu, H. Yang, C. Qiu, M. Chen, L. Hu, Y. Guo, H. Yang, C. Gu, and L. Sun, "Layer-dependent morphologies and charge transfer of Pd on n-layer graphenes," Chem Commun (Camb) 47, 9408 (2011).

31. L. M. Malard, M. A. Pimenta, G. Dresselhaus, and M. S. Dresselhaus, "Raman spectroscopy in graphene," Physics Reports 473, 51 (2009).

32. H. R. Chen, C. Y. Tsai, C. Y. Chang, K. H. Lin, C. S. Chang, and W. F. Hsieh, "Investigation of Graphene Dispersion From Kelly Sideband in Stable Mode-Locked Erbium-Doped Fiber Laser by Few-Layer Graphene Saturable Absorbers," Journal of Lightwave Technology 33, 4406 (2015). 